ARTICLE

\title{
Cathode engineering with perylene-diimide interlayer enabling over $17 \%$ efficiency single-junction organic solar cells
}

Jia Yao ${ }^{1}$, Beibei Qiu², Zhi-Guo Zhang (10 ${ }^{1 凶}$, Lingwei Xue ${ }^{1}$, Rui Wang ${ }^{3}$, Chunfeng Zhang $\mathbb{B}^{3}$, Shanshan Chen ${ }^{4,5}$,

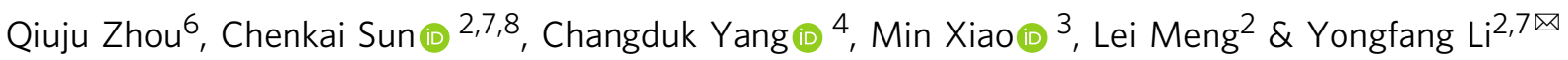

In organic solar cells (OSCs), cathode interfacial materials are generally designed with highly polar groups to increase the capability of lowering the work function of cathode. However, the strong polar group could result in a high surface energy and poor physical contact at the active layer surface, posing a challenge for interlayer engineering to address the trade-off between device stability and efficiency. Herein, we report a hydrogen-bonding interfacial material, aliphatic amine-functionalized perylene-diimide (PDINN), which simultaneously down-shifts the work function of the air stable cathodes (silver and copper), and maintains good interfacial contact with the active layer. The OSCs based on PDINN engineered silvercathode demonstrate a high power conversion efficiency of $17.23 \%$ (certified value $16.77 \%$ by NREL) and high stability. Our results indicate that PDINN is an effective cathode interfacial material and interlayer engineering via suitable intermolecular interactions is a feasible approach to improve device performance of OSCs.

\footnotetext{
${ }^{1}$ State Key Laboratory of Organic/Inorganic Composites, Beijing Advanced Innovation Center for Soft Matter Science and Engineering, Beijing University of Chemical Technology, Beijing 100029, China. ${ }^{2}$ Beijing National Laboratory for Molecular Sciences, CAS Key Laboratory of Organic Solids, Institute of Chemistry, Chinese Academy of Sciences, Beijing 100190, China. ${ }^{3}$ National Laboratory of Solid State Microstructures, School of Physics, and Collaborative Innovation Center of Advanced Microstructures, Nanjing University, Nanjing 210093, China. ${ }^{4}$ Department of Energy Engineering, School of Energy and Chemical Engineering, Low Dimensional Carbon Materials Center, Ulsan National Institute of Science and Technology (UNIST), UIsan 689-798, South Korea. ${ }^{5}$ MOE Key Laboratory of Low-grade Energy Utilization Technologies and Systems, CQU-NUS Renewable Energy Materials \& Devices Joint Laboratory, School of Energy \& Power Engineering, Chongqing University, Chongqing 400044, China. ${ }^{6}$ Analysis \& Testing Center, Xinyang Normal University, Xinyang, Henan 464000, China. ${ }^{7}$ School of Chemical Science, University of Chinese Academy of Sciences, Beijing 100049, China. ${ }^{8}$ College of Chemistry and Molecular Engineering, Zhengzhou University, Henan 450001, China. ${ }^{凶}$ email: zgzhangwhu@iccas.ac.cn; liyf@iccas.ac.cn
} 
O rganic solar cells (OSCs) have emerged as a compelling energy technology because of their advantages of simple device structure, lightweight, capability to be fabricated into flexible devices via layer-by-layer solution processing ${ }^{1,2}$. Recently, tremendous efforts are focused on designing narrow bandgap non-fullerene acceptors ${ }^{3-7}$ to overcome the intrinsic drawbacks of fullerene acceptors ${ }^{8,9}$. These efforts together with polymer donor development have promoted the power conversion efficiency (PCE) of the OSCs to a high level of $15-16 \%$ for single-junction devices ${ }^{10-15}$, demonstrating a bright future for real application of the OSCs. Besides the development of solar light harvesters ${ }^{7,16}$, interface engineering $^{17-23}$, especially cathode interlayer engineering ${ }^{24-29}$, has also played an important role for advancing the OSCs toward commercialization. However, fewer studies have aimed at cathode interlayer design in those non-fullerene-based OSCs to prevent carrier recombination at cathode and improve device stability, even though such cathode interlayer have turned out to be equally important as the active layer photovoltaic materials for developing efficient and stable OSCs (refs. ${ }^{24-29}$ ).

It has been successfully demonstrated that some alcohol/water soluble organic cathode interlayer materials (CIMs) can provide an energy-level alignment at the electrode interface, as well as an interfacial dipole for an ohmic contact ${ }^{19,30-35}$. As a typical example, we reported such effective CIMs, PDIN bearing amino group and PDINO bearing amino $\mathrm{N}$-oxide group on perylenediimide (PDI) ${ }^{36}$. Now, they are widely used by many research groups (examples are collected in Supplementary Table 2). However, for those interlayers, due to their limited ability in lowering the work function (WF) of the top electrodes; they usually work well with Al top electrode, and that is why only $\mathrm{PDINO} / \mathrm{Al}$ cathode was used to design high-efficiency non-fullerene OSCs (refs. ${ }^{10,37}$ ), tandem OSCs (ref. ${ }^{38}$ ), and even perovskite solar cells ${ }^{39}$. While the OSCs with $\mathrm{Al}$ as cathode show poor stability due to the high reactivity of $\mathrm{Al}$ electrode in air. Another concern of using Al electrode is the forming adduct with the organic interlayer during the vacuum deposit process, which will react efficiently with molecular oxygen and water ${ }^{40}$. Attempts to improve the device stability by replacing $\mathrm{Al}$ with $\mathrm{Ag}$ or $\mathrm{Cu}$ for top cathode, commonly result in a higher WF of the cathode, thus a lower open-circuit voltage $\left(V_{\mathrm{OC}}\right.$ ) of the OSCs due to a low builtin potential across the device ${ }^{26}$.

A solution is to design CIMs with highly polar groups to increase its dipole moment and thus to increase its ability in WF tunability. However, this approach is constrained by the intrinsic high surface tension of the CIMs bearing the polar group, which harms its solution deposition on active layer, and could result in poor contact between the CIM and the active layer ${ }^{26,30-34}$. It is well-known that the physically poor contact hampers electron collection by the electrode, which is one of the reasons for the low fill factor (FF) in OSCs (ref. ${ }^{41}$ ). In addition, the decohesion of the weak interface between the CIM and the active layer is inevitable for long-time device operation, and this is more severe in large area devices. In organic electronics, such as organic light-emitting diode, such interfacial issue is considered as one of the origin of the device deterioration ${ }^{42}$. Thus, it remains challenging to design CIMs that possess suitable dipole moment for decreasing WF of the air stable metals (such as Ag), and simultaneously, provide good interfacial compatibility with the active layer below and suppress carrier recombination and interlayer decohesion.

With these concerns, in this study, we develop an aliphatic amine group functionalized PDI derivative, namely PDINN, for the application as CIM in the OSCs with air stable metals Ag and $\mathrm{Cu}$ as top cathode. PDINN possesses suitable dipole moment to decrease WF of air stable metals $\mathrm{Ag}$ and $\mathrm{Cu}$ and the secondary amine in the side chains of PDINN can form hydrogen bonding with the photovoltaic materials in the active layer that results in good contact with active layer. In addition, PDINN can be facilely synthesized by a typical one-step approach under a large scale of over $64 \mathrm{~g}$ in lab from cheap raw materials, which is different from most CIMs that can only be accessible on milligram scale due to inevitable verbose multisteps synthesis and tedious purification steps. Using PDINN as cathode interlayer and $\mathrm{Ag}$ as the top cathode, OSCs with PM6 as donor and Y6 as acceptor ${ }^{10}$ (molecular structures are shown in Supplementary Fig. 1) exhibit a high PCE of $17.23 \%$ (certified PCE of $16.77 \%$ by National Renewable Energy Laboratory (NREL)). The results indicate that PDINN is a high-performance and low-cost CIM for future industrial application of the OSCs.

\section{Results}

Materials synthesis and characterization. The device structure is provided in Fig. 1a, and the photovoltaic materials (PM6 and Y6) for the active layer are shown in Supplementary Fig. 1. As shown in Fig. 1b, PDINN was easily prepared in one step, by condensation of $\mathrm{N}, \mathrm{N}$-dimethyldipropylenetriamine with perylene3,4,9,10-tetracarboxylic dianhydride in methanol. The chemical structure of PDINN was verified by mass spectrometry (Supplementary Fig. 2) and NMR spectroscopy (Supplementary Fig. 3). The reaction proceeded almost entirely in methanol with a yield of ca. 95\%. Notably, $64.4 \mathrm{~g}$ large-scale syntheses of PDINN can be readily conducted in methanol under mild reaction conditions $\left(70^{\circ} \mathrm{C}\right)$ and short reaction time $(8 \mathrm{~h})$. And the condensation reaction is convenient for further scale-up if needed. With the model for cost calculation ${ }^{43}$, the cost of material $\left(C_{\mathrm{g}}\right.$, cost-pergram) for PDINN is $1.6 \$ \mathrm{~g}^{-1}$ (Supplementary Table 1). The $C_{\mathrm{g}}$ value is less than one fifth of the well-known low-cost donor P3HT $\left(10.0 \$ \mathrm{~g}^{-1}\right)$, and less than one percent of the fullerene $\left(240 \$ \mathrm{~g}^{-1}\right)$, which is the starting material to synthesize effective fullerene derivative interlayers ${ }^{25}$. The result indicates that PDINN is really a low-cost material convenient for large-scale application.

To better understand the role of the aliphatic amine group, the thermal, physicochemical, and the photovoltaic properties of PDINN are compared with the widely used PDINO CIM. The thermal stability of PDINN and PDINO was investigated by putting the two samples on a hot plate under $150^{\circ} \mathrm{C}$ for $1 \mathrm{~h}$ followed by dissolving the samples in methanol. It can be seen that PDINO becomes insoluble in methanol, whereas, clear PDINN solution can be still obtained for the PDINN sample after the thermal treatment (Supplementary Fig. 4). The result indicates that PDINN with the aliphatic amine group is more stable than PDINO with $\mathrm{N}$-oxide amine group. The good thermal stability of PDINN is further confirmed by their thermogravimetric analysis (TGA), which show the 5\% weight loss temperature at $103.4^{\circ} \mathrm{C}$ for PDINIO and $240.1^{\circ} \mathrm{C}$ for PDINN (Supplementary Fig. 4b).

Intermolecular interactions with active layer. PDINN shows a good solubility of $26.7 \mathrm{mg} \mathrm{mL}^{-1}$ in methanol without the assistance of any acid, while most organic CIMs (such as PDIN or PFN) need hydrochloric acid or acetic acid to make a clear alcohol solution for processing 24,36 . In addition, PDINN shows a better interfacial compatibility on a wide range of active layers of OSCs compared with PDINO. For example, when a dilute solution $\left(0.5 \mathrm{mg} \mathrm{mL}^{-1}\right)$ of PDINN or PDINO was spin-coated onto the PM6:Y6 active layer at high spin speed (5000 rpm), PDINN can form a uniform film, whereas PDINO shows a poor film formation, as shown in the photo-induced force microscopy (PiFM) images in Supplementary Fig. 5. This phenomenon is likely related to the hydrogen bond formed between the secondary amine in the aliphatic amine groups of PDINN with the active layer surface. 
a

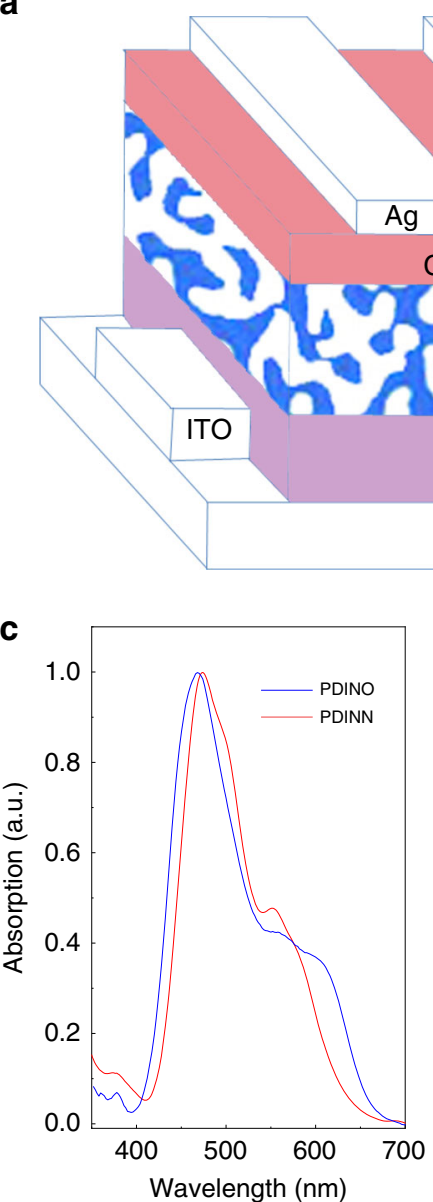

b 
Table 1 Comparison of the physicochemical properties of the CIMs of PDINO and PDINN.

\begin{tabular}{|c|c|c|c|c|c|c|c|c|}
\hline Interlayer & $\lambda_{\max }$ & $\lambda_{\text {edg }}$ & HOMO (eV) & LUMO (eV) & Conductivity $\left(10^{-5} \mathrm{~S} \mathrm{~cm}^{-1}\right)$ & Al & $\mathbf{A g}$ & $\mathbf{C u}$ \\
\hline PDINN & 473 & 630 & -6.02 & -3.78 & 50 & 3.67 & 3.72 & 4.08 \\
\hline
\end{tabular}

PDINO. The LUMO energy-level values of the PDI derivatives, especially for PDINN, are close to those of the non-fullerene acceptors $^{4,9}$, which can form a better energy-level alignment at the cathode interface and hence enhance electron collections. And the low-lying $E_{\mathrm{HOMO}}$ values of $-6.02 \mathrm{eV}$ for PDINN and $-6.21 \mathrm{eV}$ for PDINO mean that holes from various donors will be blocked sufficiently at the cathodes, with the PDI derivative CIMs. Grazing-incidence wide-angle X-ray scattering (GIWAXS) was used to measure the crystallinity of the PDI derivatives deposited on silicon substrates. As shown in Supplementary Fig. 8, both of the two PDI interlayers show a semicrystalline structure, where their defined peaks are associated with their sufficient aggregation of the PDI core in the solid for efficient charge transport, when they are used as the CIMs. With a smaller terminal group in PDINO, although it shows a stronger aggregation behavior, its predominant edge-on orientation as revealed by the strong (100) peak in the out-of-plane direction may weaken its carrier extraction ability from the active layer.

Ultraviolet photoelectron spectroscopy (UPS) was used to probe the WF values of different metal electrodes of $\mathrm{Al}, \mathrm{Ag}$, and $\mathrm{Cu}$ modified with the CIMs. The WF modification ability is frequently associated with the interfacial dipole provided by the CIMs (refs. 19,26,32,46,47). From Fig. 1e and Table 1, it can be seen that the two CIMs successfully reduced the WFs of the electrodes, the WF values of $\mathrm{Al}, \mathrm{Ag}$, and $\mathrm{Cu}$ were reduced to $3.83,3.88$, and $4.24 \mathrm{eV}$ for the PDINO CIM, and 3.67, 3.72, and $4.08 \mathrm{eV}$ for the PDINN CIM, respectively (see Table 1). PDINN shows a stronger WF lowering ability over that of PDINO for each electrode, with ca. $0.16 \mathrm{eV}$ lower WFs for the PDINN-modified electrodes than that of the PDINO-modified electrodes. It is well accepted that the dipole moment in the amine group-containing CIMs is originated from the electron transfer from the nitrogen lone pair of neutral amine group to the electrode 20,26 . Thereby, the lower WFs obtained for PDINN should be associated with its higher dipole moment from the aliphatic amine group, which is just as poly(ethyleneimine) (PEI) or its derivative does in effectively lowering the WFs of different electrodes ${ }^{20,48}$. These results encouraged us to explore PDINN as cathode interlayer with high WF metals (such as $\mathrm{Ag}$ and $\mathrm{Cu}$ ) as cathode.

The doping effects of the CIMs were investigated by the electron spin resonance (ESR) spectroscopy (Fig. 1f). PDINN shows a strong self-doping behavior ( $g$ value of 2.001) relative to PDINO ( $g$ value at 2.002), which is enabled by the unpaired electrons in aliphatic amine group of PDINN and beneficial to improving the electrical conductivity of the materials ${ }^{46,49,50}$. The electrical conductivities of the CIM films were measured by depositing two parallel silver electrodes on the two sides of the films. The conductivities of PDINO and PDINN calculated from the $I-V$ curves (Supplementary Fig. 9) are $2.4 \times 10^{-6} \mathrm{~S} \mathrm{~cm}^{-1}$ and $5.0 \times 10^{-4} \mathrm{~S} \mathrm{~cm}^{-1}$, respectively, which indicate that PDINN has a better electron-transport ability.

Photovoltaic performance. To evaluate the photovoltaic performance of the PDINN CIM in OSCs, we fabricated conventional

\begin{tabular}{|c|c|c|c|c|}
\hline Devices & $V_{o c}(V)$ & $J_{s c}\left(m A c m^{-2}\right)$ & FF (\%) & PCE (\%)b \\
\hline$\overline{w / o}$ & 0.787 & $24.98\left(24.86^{a}\right)$ & $\begin{array}{l}70.40 \\
69.51 \pm 1.81\end{array}$ & $\begin{array}{l}13.84 \\
13.39 \pm 0.53\end{array}$ \\
\hline PDINO & 0.821 & $25.58\left(24.94^{a}\right)$ & 72.24 & 15.17 \\
\hline PDINN & $\begin{array}{l}0.816 \pm 0.003 \\
0.847 \\
0.845 \pm 0.004\end{array}$ & $\begin{array}{l}25.47 \pm 0.25 \\
25.89\left(25.76^{a}\right) \\
25.51 \pm 0.28\end{array}$ & $\begin{array}{l}71.80 \pm 1.85 \\
78.59 \\
77.84 \pm 0.80\end{array}$ & $\begin{array}{l}14.94 \pm 0.42 \\
17.23 \\
16.78 \pm 0.33\end{array}$ \\
\hline PDINNC & 0.843 & 25.704 & 77.5 & 16.77 \\
\hline
\end{tabular}

devices with a structure of indium tin oxide (ITO)/PEDOT: PSS (poly (3,4-ethylenedioxythiophene): poly(styrene-sulfonate)) /PM6:Y6/ CIM/electrode. As for the active layer, PM6: Y6 blend represents the state-of-the-art system with initially reported efficiency of $15.7 \%$ by Zou, using the PDINO/Al cathode ${ }^{10}$. Recently, intensive device engineering with adding a third component in the PM6:Y6 system increased the efficiency of the ternary OSCs over $16.5 \%$ (refs. ${ }^{51-53}$ ). Without tedious component optimization, here, we demonstrate a simple yet effective cathode modification with PDINN to realize a high efficiency over 17\% for binary PM6:Y6 OSC (see Table 2). To better understand the high performance of PDINN interlayer, the device performance of PDINN was compared with that of PDINO, especially for the devices with air stable metal $\mathrm{Ag}$ and $\mathrm{Cu}$ as cathode.

Current density-voltage $(J-V)$ characteristics of the optimal OSCs without the CIM (treated with methanol), and those with the CIM are compared in Fig. 2a, and the relevant photovoltaic parameters are listed in Table 2. Figure $2 \mathrm{~b}$ shows the incident photon-to-converted current efficiency (IPCE) curves, and the integrated currents from the IPCE spectra agree quite well with the $J_{\mathrm{sc}}$ values measured from the $J-V$ curves. The PDINO/Ag device delivers a good PCE of $15.17 \%$, along with a $V_{\mathrm{oc}}$ of $0.821 \mathrm{~V}$, a $J_{\mathrm{sc}}$ of $25.58 \mathrm{~mA} \mathrm{~cm}^{-2}$, and an FF of $72.24 \%$, which is much higher than that $(13.84 \%)$ of the Ag-only device. The OSC devices with PDINN/Ag cathode demonstrated an even higher maximum PCE of $17.23 \%$, the remarkable improvement in PCE of the PDINN/Agbased device stems from the substantial increase in the key device parameters of $V_{\mathrm{oc}}$ (from $0.821 \mathrm{~V}$ to $0.847 \mathrm{~V}$ ) and $\mathrm{FF}$ (from 72.24 to $78.59 \%$ ) in comparison with the PDINO/Ag-based device. The efficiency histograms of PDINN-based and PDINO-based devices are provided in Supplementary Fig. 10. The photovoltaic performance of the optimized PDINN/Ag device was certified by NREL, and a PCE of $16.77 \%$ together with a $V_{\text {oc }}$ of $0.843 \mathrm{~V}$, an FF of $77.5 \%$, and a $J_{\mathrm{SC}}$ of $25.704 \mathrm{~mA} \mathrm{~cm}^{-2}$ was confirmed (Supplementary Fig. 11).

Interestingly, the photovoltaic performance of the PDINNbased OSCs exhibit good tolerance to the PDINN thickness variation in fabricating the OSC devices, which is important for large area fabrication of the OSCs. While the commonly used PEI 

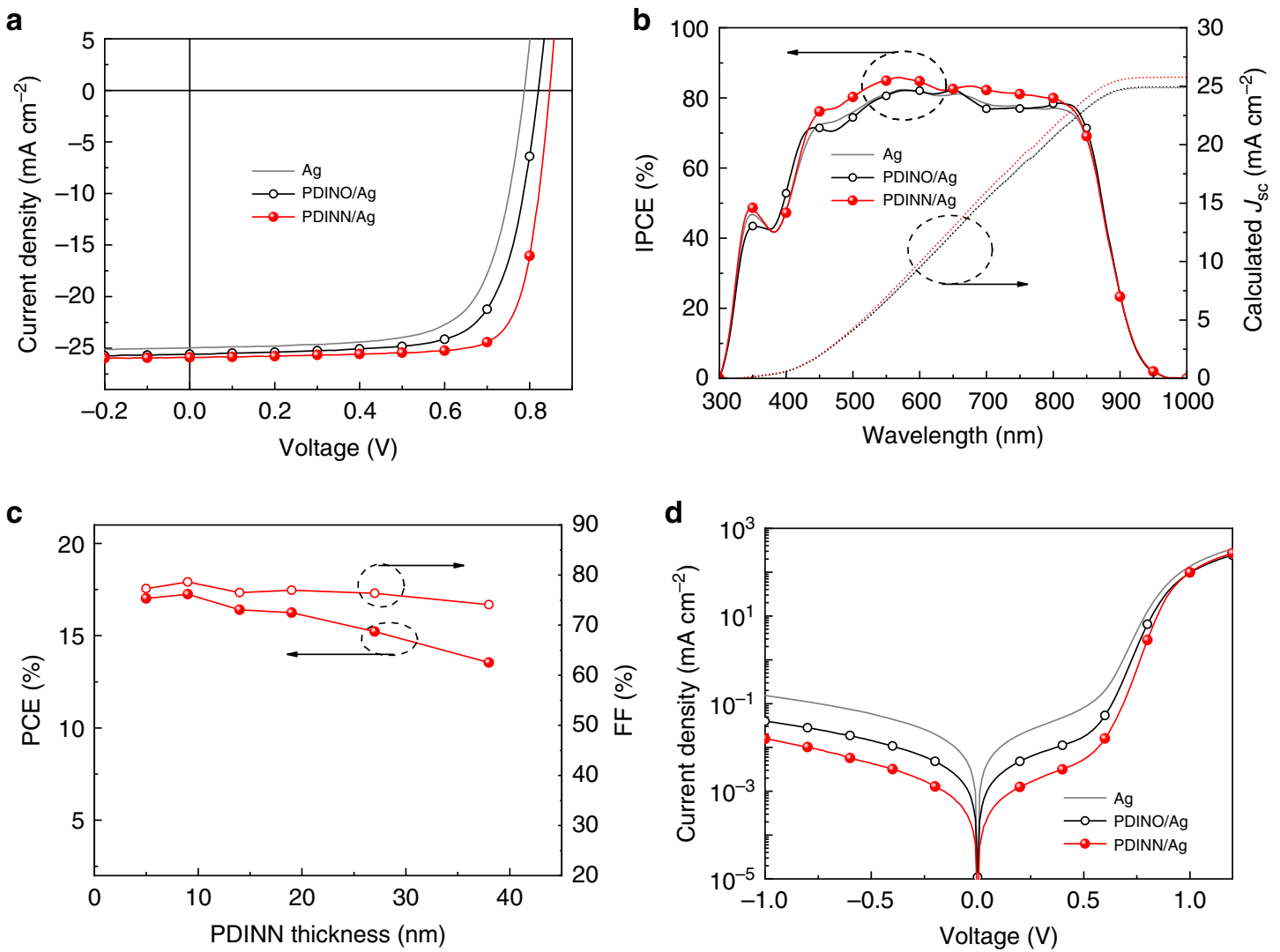

Fig. 2 Device performance of the OSCs based on PM6:Y6 with Ag as cathode. a J-V curves of the best OSCs under the illumination of AM 1.5 G, $100 \mathrm{~mW} \mathrm{~cm}^{-2}$. b IPCE spectra and integrated current density of the best devices. c Dependence of PCE and FF of the OSCs on the thickness of PDINN CIM. d Dark currents of the OSCs.

and PFN CIMs that only works well at critical thin thickness $(<5 \mathrm{~nm})^{17,26}$. For example, when the PDINN thickness rises to 27 $\mathrm{nm}$, the PCE of the devices still maintains at a high value over 15.0\% (Supplementary Fig. 12, Supplementary Table 3). Impressively, the FF still maintains a high value of $74.09 \%$ when the PDINN thickness is further increased to $38 \mathrm{~nm}$, indicating the excellent electron-transport and collection ability of the PDINN CIM. Supplementary Fig. 12c, d shows the effect of CIM thickness on the photovoltaic performance of the PDINO CIM-based OSCs. The PCE decreased from $15.46 \%$ for the optimized device with PDINO thickness of $9 \mathrm{~nm}$ to $11.72 \%$ for the OSC with PDINO thickness of $39 \mathrm{~nm}$. Notably, the PCE of the OSC with 38 nm thickness PDINN CIM is even comparable to the peak value (15.46\%) for the PDINO-based devices at its optimum thickness (9 $\mathrm{nm})$.

Based on the PDINN thickness insensitivity of the PDINN-based OSCs, we also fabricated large area devices of $1 \mathrm{~cm}^{2}$ to evaluate the prospect of the PDINN/Ag cathode in real application. The $J-V$ curve and IPCE spectrum of the large area device are plotted in Supplementary Fig. 13. As a result, a PCE value of $15.82 \%$ is obtained, along with a $V_{\text {oc }}$ of $0.850 \mathrm{~V}$, a $J_{\text {sc }}$ of $25.42 \mathrm{~mA} \mathrm{~cm}^{-2}$ (calculated $J_{\mathrm{sc}}$ from IPCE spectrum is $24.95 \mathrm{~mA} \mathrm{~cm}^{-2}$, with a mismatch of $1.85 \%$ ), and an FF of $73.19 \%$. The performance is a prominent one for the $1 \mathrm{~cm}^{2}$ device ${ }^{54}$.

The effect of CIMs on device performance. For the two CIMs, we further evaluate the charge carrier recombination behavior of the OSCs, and studied the dependence of $J_{\mathrm{sc}}$ on the light intensity $(P)$, as well as the dependence of $V_{\mathrm{oc}}$ on $P$ (ref. ${ }^{55}$ ). The dependence of $J_{\text {sc }}$ (and $V_{\text {oc }}$ ) on $P$ is shown in Fig. 3a. The relationship between $J_{\mathrm{sc}}$ and $P$ can be expressed as $J_{\mathrm{sc}} \propto P^{\alpha}$ (ref. ${ }^{56}$ ). The value of $\alpha$ should be 1 if all the free carriers were extracted by the electrode ${ }^{55}$. In our cases, the $\alpha$ value of the PDINO-based device is 0.963 , while for the PDINN-based device, its $\alpha$ value is increased to 0.982 that is very close to 1 . This means there is effective carrier collection and well-suppressed bimolecular recombination in the OSCs with PDINN as CIM. Figure 3a shows the dependence of $V_{\mathrm{oc}}$ on light intensity $P$. The slope of $V_{\mathrm{oc}}$ versus $\ln (P)$ line should be $k T / q$ for bimolecular recombination ${ }^{57}$, while the competition between bimolecular and ShockleyRead-Hall type, trap-assisted recombination makes the slope between $\mathrm{kT/q}$ and $2 \mathrm{kT} / \mathrm{q}$. The OSCs with methanol treatment (without CIM) and with PDINO or PDINN CIM exhibit the slopes of $1.42,1.24$, and $1.14 k T / q$, respectively. It is obvious that the slope of the device with PDINN CIM is closer to $k T / q$, which indicates the suppression of the trap-assisted recombination in the devices. The results collectively suggested that charge recombination was effectively suppressed in the PDINN-based OSCs.

Furthermore, we performed transient absorption (TA) measurements in a timescale up to tens of microseconds to study the effect of the CIM on the dynamics of photo-excited charges decay in the OSCs. The experimental details are described in "Methods" section. For the static transport measurements ${ }^{10}$, the average diffusion distance at a delay time of $20 \mathrm{~ns}$ can be estimated to be on the order of $10 \mathrm{~nm}$ at room temperature with no voltage applied. The diffusion length may be much longer if the timedependent local charge mobility is considered ${ }^{58,59}$. Thus for our samples, excited carrier can diffuse to the active layer surface, and the related probe can give some information on the carrier behavior that affects device performance. On the timescale of tens 

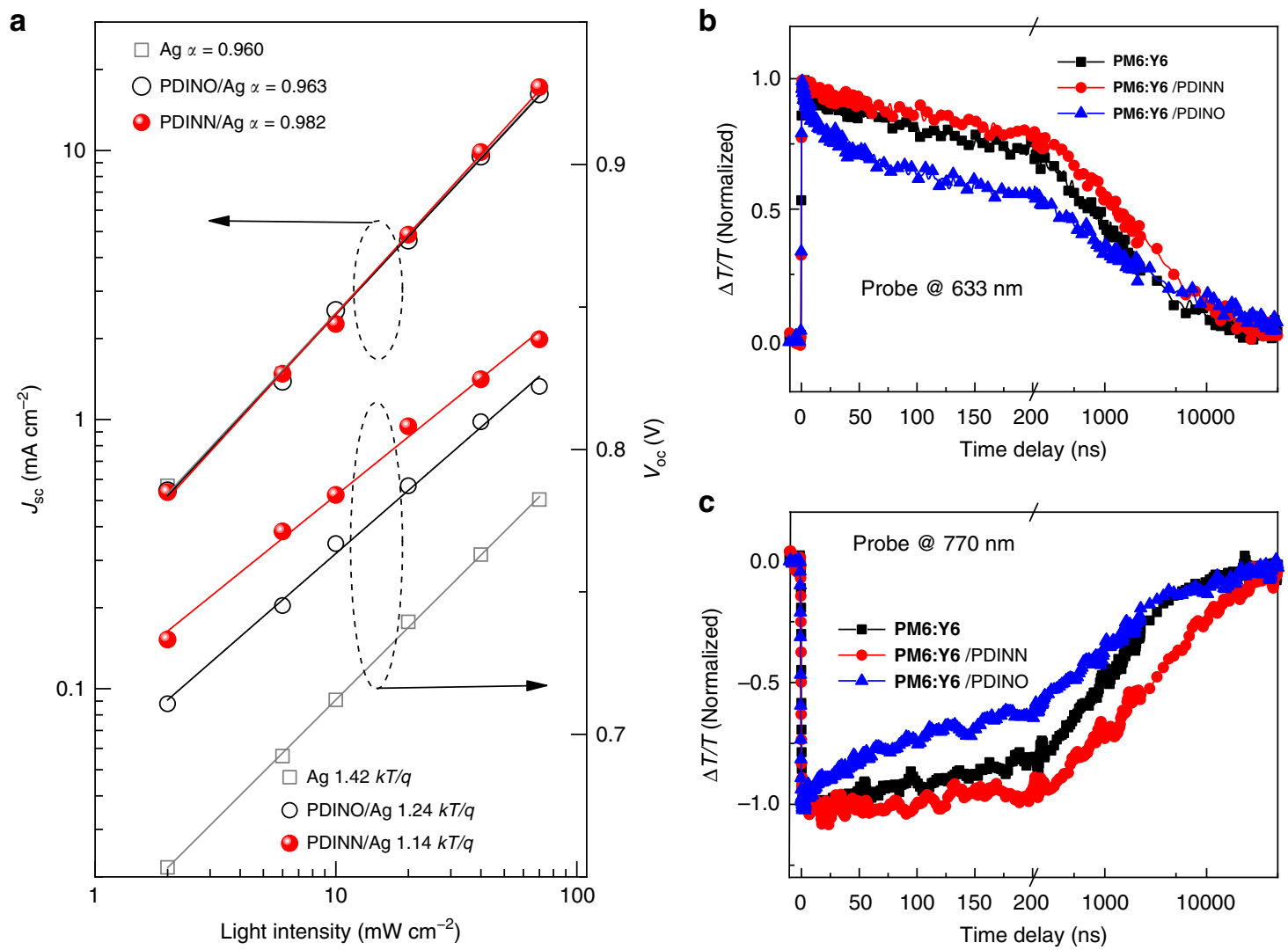

Fig. 3 The investigation of the charge carrier recombination behavior at the contact. a Light intensity dependence of $J_{\mathrm{sc}}$ and $V_{\text {oc }}$ of the OSCs based on PM6:Y6. Free charge dynamics of PM6:Y6/PDINN, PM6:Y6/PDINO, and PM6:Y6 films b at $633 \mathrm{~nm}$ probe and $\mathbf{c}$ at $770 \mathrm{~nm}$ probe, respectively.

of ns and longer, the TA data are mainly induced by the chargeseparated state of free polarons as confirmed by the photoinduced-absorption measurements (Supplementary Fig. 14). In the PM6:Y6 blend, the charge-separated state is configured with electron polaron at the acceptor site and hole polaron at the donor site, which may be captured by the excited-state absorption features of the free polarons, and the ground-state bleach features of donors and acceptors (Supplementary Fig. 14). With increasing pump fluence, the decay of free polarons becomes faster due to bimolecular recombination process (Supplementary Fig. 15). In comparison with the dynamics in the sample with PDINO, the charge recombination in the sample with PDINN is slower at the early stage (Fig. 3b, c), implying that the CIM of PDINN suppresses some loss channel.

For the different dynamics in the two CIM-treated samples, although we have no further evidence to tell how the PDI interlayers affect the charges that diffuse to the surface. The longer-lived photo-excited charges observed in PDINN-treated blend extend the charge lifetime, which is beneficial for charge collection, and thus probably responsible for the improved performance in the PDINN-treated OSCs. Typically, charge recombination is directly related with the key device parameter FF of the devices, the suppressed carrier recombination, and enhanced charge collection in the PDINN-based devices is well consistent, with its higher FF value (78.59\%) in comparison with that $(72.24 \%)$ for the PDINO-based device.

The $J-V$ characteristics of the OSCs at dark, (see Fig. 2d) show a slightly large rectification ratio thus a better diode quality for the device with PDINN CIM. To better understand the improved FF of the OSC with PDINN CIM, the electrochemical impedance spectroscopy (EIS) measurements were performed to examine the interface resistance of the devices ${ }^{60}$. Supplementary Fig. 16 shows Nyquist plots of the OSCs with PDINN or PDINO CIM at dark. A bias voltage equal to $V_{\text {oc }}$ was applied to dissipate the total current. The data were fitted using the equivalent-circuit model. The series resistances $\left(R_{\text {series }}\right)$ of the PDINN-based device $\left(0.62 \Omega \mathrm{cm}^{2}\right)$ is significantly smaller than that of the PDINObased device $\left(280.80 \Omega \mathrm{cm}^{2}\right)$. The lower series resistance should be beneficial for the higher FF of the PDINN-based devices.

Device performance with other active layers. With the successful application of PDINN CIM in the PM6:Y6-based OSCs, we further studied the universality of the PDINN CIM for the OSCs with other active layers, including PTQ10: IT-4F (ref. ${ }^{61}$ ) and J11: $m$-ITTC (ref. ${ }^{62}$; Supplementary Figs. 17 and 18). Using $\mathrm{Ag}$ as the top cathode, the direct comparison of the PCEs of the OSCs with different CIMs (PDIN/Ag in Supplementary Fig. 19) and different photoactive layers are shown in Fig. 4a, and the related photovoltaic parameters are collected in Supplementary Table 4. Clearly, the PDINN-based devices exhibit better PCEs in all the OSCs, with different active layers compared with that of the corresponding PDINO-based devices, indicating that PDINN is a universal and effective CIM for various OSCs. In consideration that all of the investigated active layers bearing halogen atom or carbonyl group, the formation of the hydrogen bond intermolecular interactions between the active layer and PDINN is also plausible. Our results also suggest that constructing intermolecular interactions between the active layer and the electrode interlayer is an effective way for further improving photovoltaic performance, and realizing application of the OSCs.

Device stability and effect of cathodes on device performance. At the optimal CIM thickness, we also explored high WF $\mathrm{Cu}$ as 
a

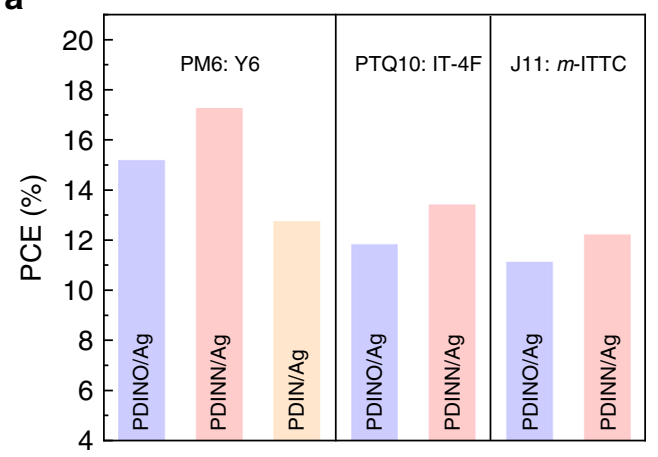

C

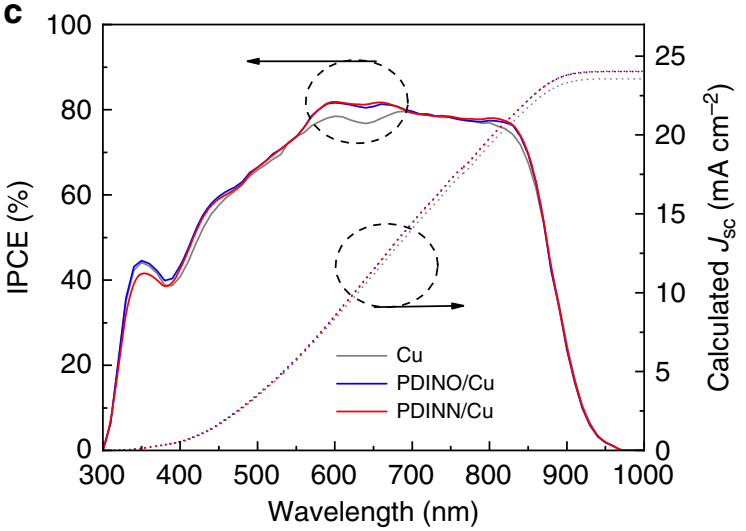

b

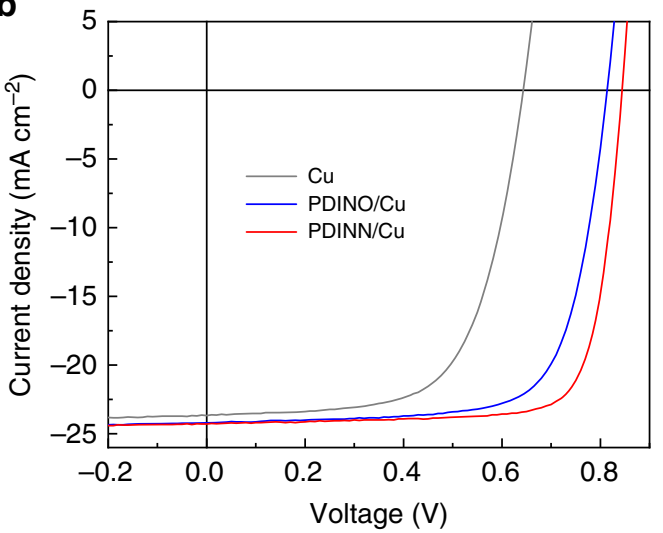

d

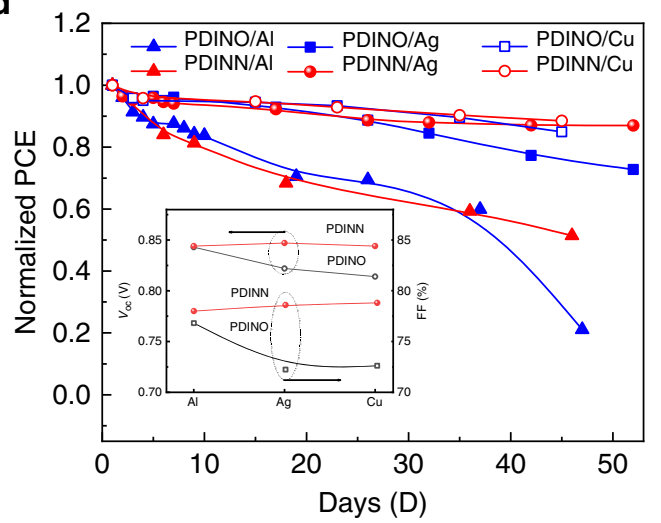

Fig. 4 Photovoltaic performance of the OSCs base on PM6:Y6. a The comparison of the PCEs of the OSCs with different CIMs and different photoactive layers under the illumination of $\mathrm{AM} 1.5 \mathrm{G}, 100 \mathrm{~mW} \mathrm{~cm}^{-2}$. b J-V curves of the best OSCs based on PM6: Y 6 blend with copper as cathode under the illumination of $A M 1.5 \mathrm{G}, 100 \mathrm{~mW} \mathrm{~cm}^{-2}$. c IPCE spectra and the integrated current density of the best devices with Cu as cathode. $\mathbf{d}$ Stability of the OSCs with different top cathode of PDINN/Ag or PDINN/Cu. The devices kept in nitrogen environment and in dark between the J-V measurements.

cathode of the OSCs based on PM6: Y6 blend to take its advantage of high conductivity and low price. The $J-V$ curves and IPCE spectra of the OSCs are shown in Fig. 4. Notably, with the PDINN CIM, $V_{\text {oc }}$ value $(0.844 \mathrm{~V})$ of the Cu-based devices is similar to that of the Ag-based devices $(0.847 \mathrm{~V})$. Impressively, a high PCE exceed $16 \%$ and a high FF of $78.8 \%$ are obtained for the $\mathrm{PDINN} / \mathrm{Cu}$-based device, indicating that $\mathrm{Cu}$ is also a good choice for cheap and stable top electrode in future commercialization of the OSCs, with PDINN as CIM. The lower IPCE value in the wavelength region from 300 to $600 \mathrm{~nm}$ is associated with the low reflectivity of the Cu-based device (Supplementary Fig. 20), which explains the slightly lower $J_{s c}$ value with $\mathrm{Cu}$ as cathode. In addition, the result suggested that a higher $J_{\text {sc }}$ value thus higher PCE could be obtained by suitable optical management ${ }^{34}$ in the $\mathrm{Cu}$ electrode-based OSCs, with PDINN as CIM.

The stability of the interlayer itself and interlayer/electrode contact is important issue for real application of OSCs. With the good air and thermal stability of PDINN, we further investigated its device stability using $\mathrm{Ag}$ or $\mathrm{Cu}$ as top electrode. After $\sim 50$ days, the PCEs of the PDINN/Ag-based and PDINN/Cubased devices can retain 87 and $88 \%$ of their initial PCE values in a glove box filled with nitrogen (Fig. 4d), while using PDINO as CIM, the PCEs remained 73 and $85 \%$ of their initial PCEs for the Ag-based and $\mathrm{Cu}$-based devices, respectively. Supplementary Fig. 21a displays the steady-state efficiency of the OSCs (in a glove box filled with nitrogen) under continuous air mass (AM) $1.5 \mathrm{G}$ $\left(100 \mathrm{~mW} \mathrm{~cm}^{-2}\right)$ illumination. The primary results over $600 \mathrm{~s}$ show a better stability of the PDINN-based devices $(98.21 \%$ of initial efficiency) than that of the PDINO-based devices (95.90\% of initial efficiency). For the unencapsulated device in air (Supplementary Fig. 21b, c), the PDINN/Ag and PDINN/Cu devices maintained $93.5 \%$ and $86.3 \%$ of their initial PCE value, whereas the PDINO/Ag and PDINO/Cu devices only showed $35.2 \%$ and $70.0 \%$ of their initial efficiency after storing $150 \mathrm{~h}$. The big difference between the stability of PDINN and PDINO-based devices is closely related to the CIMs used. The low thermal stability and moisture sensitive of PDINO can lead to an irreversible damage to the CIM-cathode contact, which renders the device stability poor. All the above results indicate that the PDINN-based OSCs show better stability than that of the PDINO-based devices.

To better understand the effect of the top electrodes $(\mathrm{Al}, \mathrm{Ag}$, and $\mathrm{Cu}$ ) and the CIMs on the device performance, corresponding device parameters are summarized in Supplementary Tables 5 and 6 for comparison ( $J-V$ curves and IPCE spectra of the Albased devices are shown in Supplementary Fig. 22). From Supplementary Table 5, it can be seen that for the PDINO-based devices with cathodes from $\mathrm{Al}, \mathrm{Ag}$ to $\mathrm{Cu}$, the $V_{\mathrm{oc}}$ values, and $\mathrm{FF}$ values are gradually decreased due to a limited WF tunability for PDINO, they are $0.843 \mathrm{~V}$ and $76.81 \%$ for $\mathrm{Al}, 0.821 \mathrm{~V}$ and $73.52 \%$ for $\mathrm{Ag}$, and $0.814 \mathrm{~V}$ and $72.61 \%$ for $\mathrm{Cu}$, respectively. In contract, the PDINN-based devices demonstrated almost unchanged $V_{\text {oc }}$ (ca. $0.844 \mathrm{~V}$ ) and FF (ca. 78.0 \%) values for all the OSCs with Al, $\mathrm{Ag}$, and $\mathrm{Cu}$ cathode (Supplementary Table 6) benefited from a strong WF tunability of PDINN. In addition, the advantage of Ag electrode with strong reflectivity and low resistance should also 
account for its higher PCE values over that of the PDINO-based OSCs. The trends of variation in $V_{\mathrm{oc}}$ and $\mathrm{FF}$ are plotted in the inset of Fig. $4 \mathrm{~d}$ for a clear comparison. The lower WF of the cathode increases the built-in electric field of the device, which is beneficial to increasing charge extraction efficiency and reducing recombination losses for higher FF of the PDINN-based OSCs. The results suggested that PDINN is an excellent CIM for future application of the OSCs with high WF stable metals as cathode.

\section{Discussion}

An aliphatic amine-functionalized PDI derivative, PDINN, is synthesized and investigated as CIM in OSCs. Compared with widely used CIM of PDINO, the advantages of PDINN are its better contact with non-fullerene active layers, stable electrode interface, higher conductivity, and stronger ability to reduce WFs of the metal cathode, which make it more suitable for use as CIM in non-fullerene OSCs with air stable metal cathode, such as Ag and $\mathrm{Cu}$, to improve the device stability. With PDINN/Ag as the top electrode, the OSCs based on PM6:Y6 exhibits a high PCE of $17.23 \%$ (certified PCE of $16.77 \%$ by NREL), which is one of the highest efficiency reported for the single-junction binary OSCs. The high efficiency is mainly benefited from its high FF of $78.59 \%$, which can be understood by the good contact with active layer and lower WF of the cathode of the OSCs. Notably, in combination with PDINN, the devices with Ag cathode not only yield higher efficiency, but also exhibit high stability. In addition, PDINN can be easily synthesized by one-step reaction with high yield and large-scale product (over $64 \mathrm{~g}$ in lab) from cheap raw materials. Thus, PDINN is a low-cost and high-performance CIM for OSCs, and is a promising CIM for future large-scale roll-toroll production and commercial application of the OSCs. Also, PDINN could also be used as cathode interlayer in other thin-film optoelectronic devices. In addition, our result also indicates that modifying the interfacial contact with suitable intermolecular interactions is a feasible approach to improve the device performance in thin-film electronic devices.

\footnotetext{
Methods

Materials and synthesis. Y6 was purchased from eFlexPV, and PM6 was purchased from Solarmer Materials.

Synthesis of PDINN: A mixture of perylene-3,4,9,10-tetracarboxylic dianhydride $(39.2 \mathrm{~g}, 100.0 \mathrm{mmol})$ and $N, N$-dimethyldipropylenetriamine $(64.1 \mathrm{~g}$, $400.0 \mathrm{mmol})$ in methanol $(300 \mathrm{~mL})$ was stirred at reflux for $8 \mathrm{~h}$ to form a clear red solution. After removal of the solvent under reduced pressure, the residue was purified by column chromatography, followed by precipitation from acetone to give PDINN as red solid (64.4 g, 95.5\%). The NMR data are in agreement with the reference ${ }^{63}$. ${ }^{1} \mathrm{H}$ NMR $\left(400 \mathrm{MHz}, \mathrm{CDCl}_{3}\right), \delta(\mathrm{ppm}): 8.40(\mathrm{~s}, 4 \mathrm{H}),, 8.23(\mathrm{t}, 4 \mathrm{H}), 4.24$ $(\mathrm{t}, 4 \mathrm{H}), 2.75(\mathrm{t}, 4 \mathrm{H}), 2.69(\mathrm{t}, 4 \mathrm{H}), 2.33(\mathrm{t}, 4 \mathrm{H}), 2.22(\mathrm{~s}, 12 \mathrm{H}), 1.96(\mathrm{t}, 4 \mathrm{H}), 1.68$ $(\mathrm{m}, 6 \mathrm{H}) .{ }^{13} \mathrm{C}$ NMR $\left(75 \mathrm{MHz}, \mathrm{CDCl}_{3}\right) \delta 162.86,133.62,130.69,128.66,125.45$ $122.86,122.55,58.01,48.30,47.31,45.56,38.65,28.40,28.18$. HRMS (MALDITOF): calcd for $\mathrm{C}_{40} \mathrm{H}_{46} \mathrm{~N}_{6} \mathrm{O}_{4}, 674.3581$, found, 674.3630
}

Material characterization. ${ }^{1} \mathrm{H}$ NMR and ${ }^{13} \mathrm{C}$ NMR spectra were recorded on Bruker AVANCE $400 \mathrm{MHz}$ or Bruker AVANCE $300 \mathrm{MHz}$ NMR spectrometer at room temperature. Mass spectra were measured on a Shimadzu spectrometer. TGA was measured on a Perkin-Elmer TGA-7 thermogravimetric analyzer with a heating rate of $10{ }^{\circ} \mathrm{C} \mathrm{min}^{-1}$ under a nitrogen flow rate of $100 \mathrm{~mL} \mathrm{~min}^{-1}$. The UV-vis absorption spectra were measured by Hitachi U-3010 UV-vis spectrophotometer. The thin-film samples were prepared by spin-coating their methanol solutions on quartz plates. To measure the thickness of thin films, several grooves were marked on the thin film by pointed tweezer, and then the depths of the grooves were recorded on Bruker DEKTAK XT step profiler. The average value of the depths was used as the thickness of thin film. Cyclic voltammetry was conducted on a Zahner IM6e electrochemical workstation using sample film coated on glassy carbon as the working electrode, Pt wire as the counter electrode, and Ag/ $\mathrm{AgCl}$ as the reference electrode, in a $0.1 \mathrm{M}$ tetrabutylammonium hexafluorophosphate $\left(\mathrm{Bu}_{4} \mathrm{NPF}_{6}\right)$ acetonitrile solution and ferrocene/ferrocenium $\left(\mathrm{Fc} / \mathrm{Fc}^{+}\right)$couple was used as an internal reference. EIS measurements were performed on Zahner IM6e electrochemical workstation, and a bias voltage equal to $V_{\mathrm{oc}}$ was applied to dissipate the total current. The UPS spectra were recorded on ESCALab250Xi multifunction X-ray photoelectron spectrometer. The ESR spectra were recorded at
$298 \mathrm{~K}$ using a JES-FA2000 ESR Spectrometer. The GIWAXS measurements were conducted at PLS-II 6 A U-SAXS beamline of the Pohang Accelerator Laboratory in Korea. The ATR FT-IR spectra was recorded on BRUKER-Fourier Transform Infrared Spectrometer-TENSOR 27. The information about contact angle and Owen method for calculating the surface energy could be found in Supplementary Method.

Morphology measurement by PiFM. The morphology of PDINN and PDINO on the active layer of PM6:Y6 was examined using PiFM (refs. ${ }^{64,65}$ ). The PiFM measurements were performed on a VistaScope AFM. Firstly, the infrared (IR) spectra of the PDINN and PDINO CIMs, and the photovoltaic materials PM6 and Y6 were measured, as shown in Supplementary Fig. 5a. Then the characteristic IR peak at $1653 \mathrm{~cm}^{-1}$ for PDINN and PDINO was selected for the PiFM morphology measurement of PDINN and PDINO films on the PM6:Y6 active layer, as shown in Supplementary Fig. 5b. Because PM6 and Y6 don't have the IR peak at $1653 \mathrm{~cm}^{-1}$, the PiFM images show only the morphology of the CIMs without the influence of PM6:Y6 active layer below it. It can be seen from Supplementary Fig. 5b, PDINO molecules aggregate and form an uneven film, while PDINN film is more uniform due to the hydrogen bonding between PDINN and the active layer.

Conductivity measurements. Solutions of PDINO and PDINN in chloroform were spin-coated at a film thickness $(T)$ of $\sim 100 \mathrm{~nm}$ on substrates with parallel silver electrodes (thickness $=100 \mathrm{~nm}$ ). The length of the electrode $(W)$ was 11,000 $\mu \mathrm{m}$ and the distance $(L)$ between the two electrodes was $500 \mu \mathrm{m}$. Electrical characterization was conducted using a PDA FS380. The conductivity was extracted from the equation of $\sigma=I \times L /(V \times W \times T)$.

Device fabrication and characterization of the OSCs. The OSCs were fabricated with a structure of ITO/PEDOT:PSS/active layer/CIM/Al, Ag or Cu. The ITO glass was cleaned by sequential ultrasonic treatment in water, deionized water, acetone and isopropanol, and then treated in an ultraviolet ozone cleaner (Ultraviolet Ozone Cleaner, Jelight Company, USA) for $20 \mathrm{~min}$. The PEDOT:PSS aqueous solution (Baytron P 4083 from H. C. Starck) was filtered through a $0.45 \mathrm{~mm}$ filter and then spin-coated on precleaned ITO-coated glass at $4000 \mathrm{rpm}$ for $30 \mathrm{~s}$. Subsequently, the PEDOT:PSS film was annealed at $150{ }^{\circ} \mathrm{C}$ for $20 \mathrm{~min}$ in air to form a $30 \mathrm{~nm}$ film. A blend solution of PM6 donor and Y6 acceptor was prepared by dissolving the materials in chloroform, and then was spin-coated at $4000 \mathrm{rpm}$ onto the PEDOT:PSS layer. Then methanol solution of CIMs (PDINO and PDINN) at a concentration of $1.0 \mathrm{mg} \mathrm{mL}^{-1}$ was deposited on the active layer at $3000 \mathrm{rpm}$ for 30 $\mathrm{s}$ to afford a cathode buffer layer. Finally, the metal cathode $\mathrm{Al}, \mathrm{Ag}$, or $\mathrm{Cu}$ was thermal evaporated under a mask at a base pressure of $\sim 10^{-5} \mathrm{~Pa}$. The photovoltaic area of the device is $4.6 \mathrm{~mm}^{2}$. Optical microscope (Olympus BX51) was used to define the active area of the devices. The $J-V$ characteristics of the OSCs were measured in a nitrogen glove box with a Keithley 2450 Source Measure unit. Oriel Sol3A Class AAA Solar Simulator (model, Newport 94023A) with a $450 \mathrm{~W}$ xenon lamp and an AM 1.5 filter was used as the light source. The light intensity was calibrated to $100 \mathrm{~mW} \mathrm{~cm}^{-2}$ by a Newport Oriel $91,150 \mathrm{~V}$ reference cell. The voltage step and delay time were $10 \mathrm{mV}$ and $1 \mathrm{~ms}$, respectively. The scan started from -1.5 $\mathrm{V}$ to $1.5 \mathrm{~V}$. The IPCE was measured by Solar Cell Spectral Response Measurement System QE-R3-011 (Enli Technology Co., Ltd., Taiwan). The light intensity at each wavelength was calibrated with a standard single-crystal Si photovoltaic cell.

TA experiment. For ns-TA experiment, the pump beam used in the experiment was emitted from a pico-second laser diode (LDH-P-C-670M, PicoQuant GmbH) at $633 \mathrm{~nm}$ or $770 \mathrm{~nm}$. The pump fluence was $\sim 1 \mu \mathrm{Jm}^{-2}$ and the carrier density could be estimated as $\sim 2 \times 10^{17} \mathrm{~cm}^{-3}$, which is close to the lowest pump condition as mentioned in the reference ${ }^{66}$. The active layers of the samples are spin-coated directly on glass substrates without top electrodes. The probe light was a supercontinuum white light generated by focusing a portion of the femtosecond laser beam from a regeneration amplifier (Libra, Coherent) onto a 3-mm-thick sapphire plate. The synchronization and time difference between the pump and probe was controlled by a digital delay generator (DG645, Stanford Instruments). The overall temporal resolution was better than $0.5 \mathrm{~ns}$. The TA signal was analyzed by an InGaAs photo-diode array (G11608, Hamamatsu) with shot to shot detection at 5 $\mathrm{kHz}$ enabled by a home-built FPGA control board. The samples were kept at nitrogen atmosphere during the measurements to avoid photodegradation.

Reporting summary. Further information on research design is available in the Nature Research Reporting Summary linked to this article.

\section{Data availability}

The data that support the findings of this study are available from the corresponding author on request.

Received: 8 December 2019; Accepted: 7 May 2020; Published online: 01 June 2020 


\section{References}

1. Yu, G., Gao, J., Hummelen, J. C., Wudl, F. \& Heeger, A. J. Polymer photovoltaic cells: enhanced efficiencies via a network of internal donoracceptor heterojunctions. Science 270, 1789-1791 (1995).

2. Li, G., Chang, W.-H. \& Yang, Y. Low-bandgap conjugated polymers enabling solution-processable tandem solar cells. Nat. Rev. Mater. 2, 17043 (2017)

3. Yan, C. et al. Non-fullerene acceptors for organic solar cells. Nat. Rev. Mater. 3, 18003 (2018).

4. Cheng, P., Li, G., Zhan, X. \& Yang, Y. Next-generation organic photovoltaics based on non-fullerene acceptors. Nat. Photonics 12, 131-142 (2018).

5. Yu, Z.-P. et al. Simple non-fused electron acceptors for efficient and stable organic solar cells. Nat. Commun. 10, 2152 (2019).

6. Swick, S. M. et al. Closely packed, low reorganization energy $\pi$-extended postfullerene acceptors for efficient polymer solar cells. Proc. Natl Acad. Sci. USA 115, E8341-E8348 (2018).

7. Chao, P. et al. A Benzo[1,2-b:4,5-c' $]$ dithiophene-4,8-dione-based polymer donor achieving an efficiency over 16\%. Adv. Mater. 32, 1907059 (2020).

8. Zhang, J., Tan, H. S., Guo, X., Facchetti, A. \& Yan, H. Material insights and challenges for non-fullerene organic solar cells based on small molecular acceptors. Nat. Energy 3, 720-731 (2018).

9. Hou, J., Inganäs, O., Friend, R. H. \& Gao, F. Organic solar cells based on nonfullerene acceptors. Nat. Mater. 17, 119 (2018).

10. Yuan, J. et al. Single-junction organic solar cell with over $15 \%$ efficiency using fused-ring acceptor with electron-deficient core. Joule 3, 1140-1151 (2019).

11. Cui, Y. et al. Over $16 \%$ efficiency organic photovoltaic cells enabled by a chlorinated acceptor with increased open-circuit voltages. Nat. Commun. 10, 2515 (2019).

12. Fan, B. et al. Achieving over $16 \%$ efficiency for single-junction organic solar cells. Sci. China Chem. 62, 746-752 (2019).

13. Jiang, K. et al. Alkyl chain tuning of small molecule acceptors for efficient organic solar cells. Joule 3, 3020-3033 (2019).

14. Liu, S. et al. High-efficiency organic solar cells with low non-radiative recombination loss and low energetic disorder. Nat. Photonics 14, 300-305 (2020).

15. $\mathrm{Wu}, \mathrm{Y}$. et al. Rationally pairing photoactive materials for high-performance polymer solar cells with efficiency of $16.53 \%$. Sci. China Chem. 63, 265-271 (2020).

16. Zhan, L. et al. Over $17 \%$ efficiency ternary organic solar cells enabled by two non-fullerene acceptors working in an alloy-like model. Energy Environ. Sci. 13, 635-645 (2020).

17. He, Z. et al. Enhanced power-conversion efficiency in polymer solar cells using an inverted device structure. Nat. Photonics 6, 591-595 (2012).

18. Small, C. E. et al. High-efficiency inverted dithienogermolethienopyrrolodione-based polymer solar cells. Nat. Photonics 6, 115-120 (2012).

19. Yip, H.-L. \& Jen, A. K. Y. Recent advances in solution-processed interfacial materials for efficient and stable polymer solar cells. Energy Environ. Sci. 5, 5994-6011 (2012).

20. Zhou, Y. et al. A universal method to produce low-work function electrodes for organic electronics. Science 336, 327-332 (2012).

21. Potscavage, W. J., Sharma, A. \& Kippelen, B. Critical interfaces in organic solar cells and their influence on the open-circuit voltage. Acc. Chem. Res. 42 , 1758-1767 (2009).

22. Lin, Y. et al. 17\% Efficient organic solar cells based on liquid exfoliated WS2 as a replacement for PEDOT:PSS. Adv. Mater. 31, 1902965 (2019).

23. Liu, L. et al. Graphdiyne derivative as multifunctional solid additive in binary organic solar cells with $17.3 \%$ efficiency and high reproductivity. Adv. Mater. 32, 1907604 (2020)

24. He, Z. et al. Simultaneous enhancement of open-circuit voltage, short-circuit current density, and fill factor in polymer solar cells. Adv. Mater. 23, 4636-4643 (2011).

25. Lai, Y.-Y., Cheng, Y.-J. \& Hsu, C. S. Applications of functional fullerene materials in polymer solar cells. Energy Environ. Sci. 7, 1866-1883 (2014).

26. Page, Z. A., Liu, Y., Duzhko, V. V., Russell, T. P. \& Emrick, T. Fulleropyrrolidine interlayers: tailoring electrodes to raise organic solar cell efficiency. Science 346, 441-444 (2014).

27. Liao, S.-H., Jhuo, H.-J., Cheng, Y.-S. \& Chen, S.-A. Fullerene derivative-doped zinc oxide nanofilm as the cathode of inverted polymer solar cells with lowbandgap polymer (PTB7-Th) for high performance. Adv. Mater. 25, 4766-4771 (2013).

28. Zhang, F., Ceder, M. \& Inganäs, O. Enhancing the photovoltage of polymer solar cells by using a modified cathode. Adv. Mater. 19, 1835-1838 (2007).

29. Nian, L. et al. Photoconductive cathode interlayer for highly efficient inverted polymer solar cells. J. Am. Chem. Soc. 137, 6995-6998 (2015).

30. Duan, C., Zhang, K., Zhong, C., Huang, F. \& Cao, Y. Recent advances in water/alcohol-soluble [small pi]-conjugated materials: new materials and growing applications in solar cells. Chem. Soc. Rev. 42, 9071-9104 (2013).
31. Liu, Y. et al. Transforming ionene polymers into efficient cathode interlayers with pendent fullerenes. Angew. Chem. Int. Ed. 58, 5677-5681 (2019).

32. Ouyang, X., Peng, R., Ai, L., Zhang, X. \& Ge, Z. Efficient polymer solar cells employing a non-conjugated small-molecule electrolyte. Nat. Photonics $\mathbf{9}$, 520-524 (2015)

33. Oh, S.-H. et al. Water-soluble polyfluorenes as an interfacial layer leading to cathode-independent high performance of organic solar cells. Adv. Funct. Mater. 20, 1977-1983 (2010).

34. Steim, R., Kogler, F. R. \& Brabec, C. J. Interface materials for organic solar cells. J. Mater. Chem. 20, 2499-2512 (2010).

35. Seo, J. H. et al. Improved high-efficiency organic solar cells via incorporation of a conjugated polyelectrolyte interlayer. J. Am. Chem. Soc. 133, 8416-8419 (2011).

36. Zhang, Z.-G. et al. Perylene diimides: a thickness-insensitive cathode interlayer for high performance polymer solar cells. Energy Environ. Sci. 7, 1966-1973 (2014)

37. Lin, Y. et al. An electron acceptor challenging fullerenes for efficient polymer solar cells. Adv. Mater. 27, 1170-1174 (2015).

38. Meng, L. et al. A tandem organic solar cell with PCE of $14.52 \%$ employing subcells with the same polymer donor and two absorption complementary acceptors. Adv. Mater. 31, e1804723 (2019).

39. Hou, Y. et al. Overcoming the interface losses in planar heterojunction perovskite-based solar cells. Adv. Mater. 28, 5112-5120 (2016).

40. Jørgensen, M. et al. Stability of polymer solar cells. Adv. Mater. 24, 580-612 (2012).

41. Guillemoles, J.-F., Kirchartz, T., Cahen, D. \& Rau, U. Guide for the perplexed to the Shockley-Queisser model for solar cells. Nat. Photonics 13, 501-505 (2019).

42. Kato, S. \& Pac, C. Molecularly thin polymer films that function to enhance charge injection efficiency in organic light-emitting diodes. J. Phys. Chem. B 108, 19932-19939 (2004)

43. Li, X. et al. Simplified synthetic routes for low cost and high photovoltaic performance n-type organic semiconductor acceptors. Nat. Commun. 10, 519 (2019).

44. Sukhishvili, S. A. \& Granick, S. Layered, erasable, ultrathin polymer films. $J$. Am. Chem. Soc. 122, 9550-9551 (2000).

45. Wagner, W., Wehner, M., Stepanenko, V., Ogi, S. \& Würthner, F. Living supramolecular polymerization of a perylene bisimide dye into fluorescent Jaggregates. Angew. Chem. Int. Ed. 56, 16008-16012 (2017).

46. $\mathrm{Wu}, \mathrm{Z}$. et al. $\mathrm{n}$-Type water/alcohol-soluble naphthalene diimide-based conjugated polymers for high-performance polymer solar cells. J. Am. Chem. Soc. 138, 2004-2013 (2016).

47. Kang, H., Hong, S., Lee, J. \& Lee, K. Electrostatically self-assembled nonconjugated polyelectrolytes as an ideal interfacial layer for inverted polymer solar cells. Adv. Mater. 24, 3005-3009 (2012).

48. Liu, Y., Duzhko, V. V., Page, Z. A., Emrick, T. \& Russell, T. P. Conjugated polymer zwitterions: efficient interlayer materials in organic electronics. Acc. Chem. Res. 49, 2478-2488 (2016).

49. Tang, C. G. et al. Doped polymer semiconductors with ultrahigh and ultralow work functions for ohmic contacts. Nature 539, 536-540 (2016).

50. Li, C.-Z. et al. Doping of fullerenes via anion-induced electron transfer and its implication for surfactant facilitated high performance polymer solar cells. Adv. Mater. 25, 4425-4430 (2013).

51. An, Q., Ma, X., Gao, J. \& Zhang, F. Solvent additive-free ternary polymer solar cells with $16.27 \%$ efficiency. Sci. Bull. 64, 504-506 (2019).

52. Gao, J. et al. Over $16.7 \%$ efficiency of ternary organic photovoltaics by employing extra PC71BM as morphology regulator. Sci. China Chem. 63, 83 (2020).

53. Chang, Y. et al. The synergy of host-guest nonfullerene acceptors enables $16 \%$-efficiency polymer solar cells with increased open-circuit voltage and fillfactor. Mater. Horiz. 6, 2094-2102 (2019).

54. Fan, B. et al. Fine-tuning of the chemical structure of photoactive materials for highly efficient organic photovoltaics. Nat. Energy 3, 1051-1058 (2018).

55. Cowan, S. R., Roy, A. \& Heeger, A. J. Recombination in polymer-fullerene bulk heterojunction solar cells. Phys. Rev. B 82, 245207-245217 (2010).

56. Kyaw, A. K. et al. Intensity dependence of current-voltage characteristics and recombination in high-efficiency solution-processed small-molecule solar cells. ACS Nano 7, 4569-4577 (2013).

57. Cowan, S. R., Roy, A. \& Heeger, A. J. Recombination in polymer-fullerene bulk heterojunction solar cells. Phys. Rev. B 82, 245207 (2010).

58. Burke, T. M. \& McGehee, M. D. How high local charge carrier mobility and an energy cascade in a three-phase bulk heterojunction enable $>90 \%$ quantum efficiency. Adv. Mater. 26, 1923-1928 (2014).

59. Pranculis, V. et al. Charge carrier generation and transport in different stoichiometry APFO3:PC61BM solar cells. J. Am. Chem. Soc. 136, $11331-11338$ (2014) 
60. Liao, Q. et al. Tailoring and modifying an organic electron acceptor toward the cathode interlayer for highly efficient organic solar cells. Adv. Mater. 32, 1906557 (2019).

61. Sun, C. et al. A low cost and high performance polymer donor material for polymer solar cells. Nat. Commun. 9, 743 (2018).

62. Qiu, B. et al. A simple approach to prepare chlorinated polymer donors with low-lying HOMO level for high performance polymer solar cells. Chem. Mater. 31, 6558-6567 (2019).

63. Zhao, L. et al. Layer-by-layer deposited multilayer films of oligo(pyrenebutyric acid) and a perylene diimide derivative: structure and photovoltaic properties. Langmuir 24, 4380-4387 (2008).

64. Jahng, J. et al. Linear and nonlinear optical spectroscopy at the nanoscale with photoinduced force microscopy. Acc. Chem. Res. 48, 2671-2679 (2015).

65. Gu, K. L. et al. Nanoscale domain imaging of all-polymer organic solar cells by photo-induced force microscopy. ACS Nano 12, 1473-1481 (2018).

66. Howard, I. A., Mauer, R., Meister, M. \& Laquai, F. Effect of morphology on ultrafast free carrier generation in polythiophene:fullerene organic solar cells. J. Am. Chem. Soc. 132, 14866-14876 (2010).

\section{Acknowledgements}

The work was supported by the National Natural Science Foundation of China (nos. 51722308, 51673200, 21734008, and 51820105003), Guangdong Major Project of Basic and Applied Basic Research (No. 2019B030302007), Fundamental Research Funds for the Central Universities (Buctrc201822 and XK1802-2), and the Key Research Programs in Universities of Henan Province (18A150049). The authors thank Prof. Wang Xiuyu and Dr. Zhang Jinyuan for their valuable discussions.

\section{Author contributions}

Y.F.L. and Z.G.Z. supervised the project. Z.G.Z. designed PDINN, Z.G.Z. and L.W.X. synthesized PDINN and PDINO, L.W.X. characterized the chemical structure of PDINN and PDINO, J.Y. and B.B.Q. carried out the device fabrication and characterization, L.M. analyzed the device parameters, S.S.C. and C.Y. measured the GIWAXS diffraction patterns, Q.J.Z. confirmed the H-bonding material of PDINN, C.K.S. synthesized PTQ10,
R.W., C.F.Z., and M.X. measured TA spectra, and provide discussions. Z.G.Z., J.Y., and Y.F.L. wrote the paper.

\section{Competing interests}

The authors declare no competing interests.

\section{Additional information}

Supplementary information is available for this paper at https://doi.org/10.1038/s41467020-16509-w.

Correspondence and requests for materials should be addressed to Z.-G.Z. or Y.L.

Peer review information Nature Communications thanks the anonymous reviewer(s) for their contribution to the peer review of this work. Peer reviewer reports are available.

Reprints and permission information is available at http://www.nature.com/reprints

Publisher's note Springer Nature remains neutral with regard to jurisdictional claims in published maps and institutional affiliations.

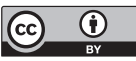

Open Access This article is licensed under a Creative Commons Attribution 4.0 International License, which permits use, sharing, adaptation, distribution and reproduction in any medium or format, as long as you give appropriate credit to the original author(s) and the source, provide a link to the Creative Commons license, and indicate if changes were made. The images or other third party material in this article are included in the article's Creative Commons license, unless indicated otherwise in a credit line to the material. If material is not included in the article's Creative Commons license and your intended use is not permitted by statutory regulation or exceeds the permitted use, you will need to obtain permission directly from the copyright holder. To view a copy of this license, visit http://creativecommons.org/ licenses/by/4.0/.

(C) The Author(s) 2020 